\title{
Neumomediastino espontáneo (síndrome de Hamman): U na enfermedad benigna mal diagnosticada
}

\section{Carlos Álvarez $\mathrm{Z}^{1,2}$, Andrés Jadue Ta, Francisco Rojas $\mathrm{R}^{\mathrm{a}}$, César Cerda $\mathrm{C}^{\mathrm{b}}$, Miguel Ramírez Vc, Carlos Cornejo $\mathrm{S}^{2}$. \\ Spontaneous pneumomediastinum. Review of eight cases}

Background: Hamman syndrome or spontaneous pneumomediastinum is uncommon and its clinical manifestations are chest pain, dyspnea and subcutaneous emphysema. Aim: To report a series of patients with spontaneous pneumomediastinum. Material and methods: Medical records of patients with the diagnosis of pneumomediastinum, managed between 2002 and 2007 in a public hospital, were retrieved and reviewed. Results: Eight patients aged between 16 and 41 years (five males) were identified. The most common symptom was chest pain and the most common sign was subcutaneous emphysema. A chest X ray was performed in all and a chest CT scan in seven. All were managed conservatively with oxygen, analgesia and rest. No patient required surgery and the evolution was favorable. Conclusions: The most common presenting complaint of spontaneous pneumomediastinum is chest pain and its management does not require surgery (Rev Méd Chile 2009; 137: 1045-50).

(Key words: Chest pain; Mediastinal emphysema; Subcutaneous emphysema)

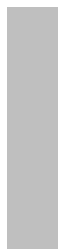

\footnotetext{
Recibido el 20 de mayo, 2009. Aceptado el 27 de julio, 2009.

${ }^{1}$ Departamento de Cirugía, Facultad de Ciencias Médicas, Universidad de Santiago de Chile (USACH). ${ }^{2}$ Equipo de Cirugía de Tórax, Servicio de Cirugía Complejo Asistencial Barros Luco (CABL).

${ }^{a}$ Médico Becario de Cirugía, Universidad de Chile.

bMédico Becario de Cirugía, Universidad de Valparaíso.

${ }^{\mathrm{c}}$ Médico General de Zona Dirección de Salud Rural de Aysén.
}

E síndrome de Hamman o neumomediastino Cespontáneo (NME) descrito por primera vez por Louis Hamman en 1939 como "enfisema mediastínico espontáneo", corresponde a la presencia de aire libre en el mediastino que se

Correspondencia a: Dr. Carlos Alvarez Zepeda. Salmos Sur 107, Las Palmas de Maipú. Maipú, Santiago, Chile. Fono/ Fax: 551-4320. E mail: doctorcmaz@gmail.com presenta sin relación a trauma torácico, procedimientos traqueobronquiales o esofágicos, ventilación mecánica, cateterización cardiaca ni cirugía torácica $^{1-3}$. Es una enfermedad poco frecuente que se diagnostica en 1/7.000 a 1/44.000 ingresos hospitalarios y suele ocurrir en pacientes jóvenes sin comorbilidad aparente, con excepción del $\operatorname{asma}^{4,5}$

Las manifestaciones clínicas más frecuentes son dolor torácico, disnea y enfisema subcutáneo ${ }^{5-8}$. 
Hamman describió además un signo muy característico, pero no tan frecuente, que consiste en la crepitación que ocurre concomitantemente con el latido cardiaco y que se percibe en la auscultación de la región anterior del tórax (signo de Hamman) ${ }^{1}$.

El NME es una enfermedad de evolución generalmente benigna, y que suele no diagnosticarse acertadamente cuando se presenta9-11.

Los cirujanos de tórax se ven involucrados en el diagnóstico y manejo de esta entidad, con el fin de excluir en forma cierta una condición grave que pudiese comprometer la vida y requiriera un tratamiento urgente, como el neumomediastino secundario a rotura traqueobronquial o perforación esofágica, la mediastinitis o el neumotórax ${ }^{4,9}$.

El objetivo de este trabajo es presentar un grupo de pacientes con NME o síndrome de Hamman atendidos en el Equipo de Cirugía de Tórax del Servicio de Cirugía del Complejo Asistencial Barros Luco (CABL) desde enero de 2002 hasta abril de 2007 y describir las características clínicas, los exámenes complementarios, el manejo, la evolución y seguimiento, y entregar una revisión actualizada del tema.

\section{MATERIALES Y MÉTODOS}

Se realizó un estudio retrospectivo, descriptivo. Se seleccionaron desde los registros computacionales de la base de datos de pacientes del Equipo de Cirugía de Tórax del CABL (todos mayores de 14 años), los casos cuyo diagnóstico de egreso fue NME entre enero de 2002 y abril de 2007. Para el diagnóstico de NME los pacientes cumplieron con los siguientes criterios: la presencia de al menos un examen radiológico que fuera consistente con neumomediastino, la ausencia de un evento traumático que explicara el cuadro y la ausencia de una enfermedad pulmonar cuyo sustrato anatómico justificara el aire en el mediastino (por ej: enfisema pulmonar, bulas congénitas). Se excluyeron todos los pacientes con diagnóstico de neumomediastino que tuvieron un hecho desencadenante bien identificado, tal como perforación esofágica o del árbol traqueobronquial, cirugía cardiaca o torácica reciente, infecciones por gérmenes productores de gas o cualquier enfermedad que involucrara el cuello o el abdomen. De la ficha médica se registraron los antece- dentes remotos, la presencia de algún factor desencadenante, las manifestaciones clínicas y las pruebas complementarias. Se precisó el manejo y la evolución de cada caso. Se realizó seguimiento telefónico de todos los pacientes.

Para la revisión bibliográfica se realizó una búsqueda a través del Catálogo Bello, LILACS, Pubmed y Scielo sin restricción de tiempo o idioma.

\section{Resultados}

Se identificaron 8 pacientes $(5$ hombres y 3 mujeres). La edad promedio fue de 25 años. Cuatro de los pacientes presentaban tabaquismo activo, 3 pacientes eran consumidores de cocaína y 3 pacientes tenían diagnóstico previo de asma.

Al estudiar los posibles factores precipitantes asociados con el episodio agudo de NME encontramos que: un paciente presentó tos frecuente en relación a cuadro de bronquitis aguda, un paciente sufrió inhalación de irritante (ortoclorobenzalmalanonitrilo, compuesto de bomba lacrimógena), 3 pacientes inhalaron cocaína y 3 tuvieron crisis asmática (Tabla 1).

El tiempo promedio de consulta desde el inicio de los síntomas fue de 20 h. El síntoma más frecuente fue el dolor torácico (6 pacientes), seguido de la autopercepción del enfisema subcutáneo toracocervical (3 pacientes) y la disnea (3 pacientes). Seis de ocho pacientes presentaron enfisema subcutáneo durante el examen físico. En 2 pacientes se pesquisó signo de Hamman. Todos los pacientes fueron estudiados con Rx de tórax la cual evidenció la presencia de aire libre en el mediastino. Siete pacientes fueron estudiados además con TC de tórax (Figura 1). A 6 pacientes se les realizó exámenes de laboratorio, los cuales no presentaron alteraciones significativas. Un paciente fue sometido además a esofagograma de emergencia con el fin de descartar una lesión esofágica, ya que se había sometido a una endoscopia digestiva alta una semana antes del inicio de los síntomas, con el fin de estudiar un cuadro úlcero-péptico de larga data. El mismo paciente en la anamnesis próxima refirió inhalación de cocaína en concomitancia con el episodio agudo de NME.

Sólo uno de los 8 pacientes presentó como diagnóstico de ingreso NME (Tabla 2). Los otros 
Tabla 1. Tabla paciente y factores precipitantes presentes

\begin{tabular}{|cccl|}
\hline Paciente N 0 & Sexo & Edad (años) & Factor precipitante* \\
\hline 1 & M & 16 & Crisis asmática \\
2 & $\mathrm{~F}$ & 19 & Tos frecuente por bronquitis aguda \\
3 & $\mathrm{~F}$ & 19 & Crisis asmática \\
4 & $\mathrm{M}$ & 16 & Inhalación humo de bomba lacrimógena \\
5 & $\mathrm{M}$ & 55 & Consumo de cocaína \\
6 & $\mathrm{M}$ & 41 & Consumo de cocaína \\
7 & $\mathrm{~F}$ & 20 & Crisis asmática \\
8 & $\mathrm{M}$ & 16 & Consumo de cocaína \\
\hline
\end{tabular}

M: masculino; F: femenino.

*Factor rescatado en la historia clínica en relación temporal con el cuadro de NME.

Tabla 2. Tabla paciente y diagnóstico de ingreso

\begin{tabular}{|cccl|}
\hline Paciente N 0 & Edad (años) & Sexo & D iagnóstico de ingreso* \\
\hline 1 & 16 & M & Neumomediastino espontáneo \\
2 & 19 & F & Ruptura de vía aérea \\
3 & 19 & F & Neumotórax espontáneo \\
4 & 16 & M & Dolor torácico agudo en estudio \\
5 & 55 & M & Enfisema subcutáneo en estudio \\
6 & 41 & M & Mediastinitis \\
7 & 20 & F & Neumotórax espontáneo \\
8 & 16 & M & Perforación esofágica \\
\hline
\end{tabular}

M: masculino; F: femenino.

*Diagnóstico rescatado de la ficha de ingreso a la Unidad de Emergencia.

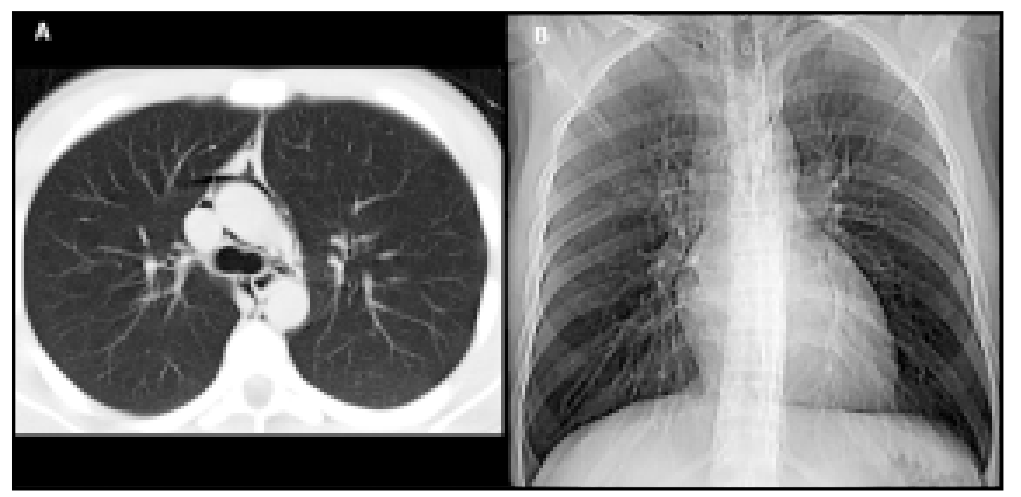

Figura 1. A: TC de tórax donde se observa aire separando estructuras mediastínicas. B: radiografía PA de tórax donde se observa aire alrededor del corazón y grandes vasos, además del signo de diafragma continuo.

diagnósticos fueron neumotórax, rotura de vía aérea, dolor torácico, perforación esofágica y mediastinitis.

Todos los pacientes fueron tratados con reposo, analgesia y oxigenoterapia. Tres pacientes requirieron además tratamiento de su crisis asmá- tica (hidrocortisona intravenosa, salbutamol en inhalación). Ningún paciente requirió cirugía.

La evolución fue favorable en todos los pacientes con un promedio de estadia hospitalaria de 3 días. Todos se controlaron en policlínica de 
cirugía a la semana y se encontraban en perfectas condiciones. En el seguimiento telefónico, que se completó en todos los pacientes (con un promedio de 30 meses), se pesquisó una recidiva al año del alta, la cual fue tratada también en forma conservadora con éxito.

\section{DisCUSIÓN}

El neumomediastino o la presencia de aire en el mediastino fue descrito por primera vez por Laennec en 1819 en relación al trauma ${ }^{12}$. No obstante en la literatura médica se encuentra un caso de enfisema subcutáneo posparto reportado por Simmons ya en 1783, más probablemente el primer caso de NME del que se tenga conocimiento $^{13}$. Sin embargo, no fue sino hasta 1939 que Hamman informa los primeros casos de enfisema subcutáneo asociado a neumomediastino, fecha con la que además aparece el término "síndrome de Hamman"1,9,14. En 1944, Macklin y Macklin describieron sus bases fisiopatológicas, las que consisten en la rotura de alvéolos terminales, con la consiguiente liberación de aire al intersticio pulmonar, el cual migraría por la vaina de los vasos del hilio pulmonar al mediastino ${ }^{15,16}$. Finalmente el aire puede extenderse hacia los tejidos subcutáneos de la pared torácica y del cuello (enfisema subcutáneo), siguiendo los planos fasciales y eventualmente hacia el canal raquídeo (neumatosis epidural o neumorraquis) ${ }^{17,18}$.

La enfermedad tiene una incidencia no bien establecida, pero aparentemente baja ${ }^{4-10}$. Al igual que Macia y cols, pensamos que probablemente es una entidad subdiagnosticada, ya que se conoce poco, sus síntomas no son específicos y tiene signos radiológicos difíciles de identificar ${ }^{5}$. En nuestra serie sólo se hizo el diagnóstico al ingreso en 1 de los 8 casos (12\%), a pesar que siempre presentaron el cuadro clínico característico y los antecedentes típicos y que ninguno tenía hallazgos de una enfermedad grave.

La literatura médica recoge la presentación de casos aislados o series pequeñas, la mayor de ellas alcanza a los 41 enfermos $^{3-11}$. En Chile encontramos dos recientes publicaciones que comunican dos y un caso, respectivamente ${ }^{19,20}$.

El NME afecta principalmente a adultos jóvenes. Más de 75\% de los casos informados son hombres con un promedio de edad de 20 años $5,8,9,21,22$. En nuestros casos 5 de 8 fueron varones y la edad promedio fue de 25 años.

Se han descrito factores desencadenantes de la rotura alveolar que llevarían al NME, todos los cuales incrementarían la presión endopulmonar. Entre éstos se encuentran los ataques de tos, como en las crisis asmáticas, los vómitos, como en los casos descritos de cetoacidosis diabética, o los autoinducidos de la anorexia nerviosa ${ }^{23-26}$. Las maniobras de Valsalva, como en el trabajo de parto y la defecación ${ }^{27,28}$. La inhalación profunda de drogas ilícitas (cocaína, marihuana, éxtasis) ${ }^{29-32}$. En pacientes sometidos a espirometría durante la maniobra del volumen espiratorio forzado (FEV) y en individuos sanos durante actividades deportivas $^{33,36}$. En nuestros casos el factor desencadenante más asociado fue el antecedente de asma y la inhalación de cocaína.

Además de los clásicos síntomas y signos ya mencionados de dolor torácico, disnea y enfisema subcutáneo, se han descrito en otras series tortícolis, odinofagia, disfagia, dolor cervical, cambios en el tono de la voz o disfonía y pulso paradojal ${ }^{19,35,36}$. En nuestros casos 6 de los 8 pacientes presentaron dolor torácico, 3 de los 8 disnea y 6 enfisema subcutáneo cervical o torácico (Tabla 3).

Ante la sospecha, el diagnóstico se confirma con la radiografía de tórax. Los signos que se evidencian son la presencia de aire separando estructuras mediastínicas o rodeándolas, el enfisema subcutáneo y el aire subesternal (Tabla 4) ${ }^{37}$. La TC de tórax se ha demostrado superior a la Rx de tórax, tanto en el diagnóstico, localización y extensión, como en el diagnóstico diferencial ${ }^{38}$.

En la práctica vemos que en nuestros casos pocas veces se sospechó el diagnóstico, la radiografía fue parte del estudio del dolor torácico y una vez encontrado el aire en el mediastino, tan sólo una vez fue acertada la conclusión, en las otras se pensó siempre en una causa más grave de su origen y el paciente fue sometido a TC de tórax o incluso en uno se realizó un estudio baritado de esófago.

El diagnóstico diferencial debe hacerse con otras causas de dolor torácico, en especial con aquellas que presenten enfisema subcutáneo asociado, como la perforación esofágica espontánea (síndrome de Boerhaave) y el neumotórax espontáneo. Ambas 
Tabla 3. Tabla frecuencia de síntomas y signos

\begin{tabular}{|lclc|}
\hline Síntoma & Frecuencia & Signo & Frecuencia \\
\hline Dolor torácico & 6 & ES en el tórax & 4 \\
Disnea & 3 & ES en el cuello & 4 \\
Dolor cervical & 2 & Signo de Hamman & 2 \\
Disfagia & 2 & Aumento de V faríngeo & 1 \\
Disfonía & 2 & & \\
Odinofagia & 1 & & \\
Tos & 1 & & \\
\hline
\end{tabular}

ES: enfisema subcutáneo. V: volumen.

Tabla 4. Tabla signos radiológicos de neumomediastino*

Enfisema subcutáneo

Signo de la vela tímica

Neumopericardio

Signo del anillo alrededor de la arteria

Signo de la arteria tubular

Signo de la pared bronquial doble

Signo del diafragma continuo

Aire en el ligamento pulmonar

Aire extrapleural

*Zylak CM, Standen JR, Barnes GR, Zylak CJ. Pneumomediastinum revisited. RadioGraphics 2000; 20: $1043-57$.

entidades potencialmente de mucha mayor gravedad y de eventual resolución quirúrgica.

El curso clínico del NME está ampliamente enunciado en los reportes clínicos publicados y también en nuestra serie; es en general benigno y autolimita-

\section{REFERENCIAS}

1. Hamman L. Spontaneous mediastinal emphysema. Bull Johns Hopkins Hospital 1939; 64: 1-21.

2. PIERSON DJ. Pneumomediastinum. In: Murray JF, Nadel JA, Eds. Textbook of respiratory medicine. $2^{\text {nd }}$ Ed. Philadelphia: WB Saunders, 1994; 2250-65.

3. BoDEY GP. Medical mediastinal emphysema. Ann Intern Med 1961; 54: 46-56.

4. Gerazounis M, Athanassiadi K, Kalantzi N, Moustardas M. Spontaneous pneumomediastinum: a rare benign entity. J Thorac Cardiovasc Surg 2003; 126: 774-6. $\mathrm{do}^{5,6,9}$. La recidiva reportada es poco frecuente $\mathrm{e}^{21,36}$. Uno de nuestros casos presentó un nuevo episodio al año de seguimiento y también fue de evolución favorable. La resolución clínica completa se produce no más allá del cuarto día. El tratamiento es sintomático y habitualmente consiste en analgesia, oxigenoterapia y reposo. Al igual que en los casos comunicados en la literatura nuestra serie no requirió de un procedimiento quirúrgico ${ }^{10,22,36}$.

En conclusión, el síndrome de Hamman o NME es un desafío diagnóstico para el médico de emergencia y el cirujano de tórax. Su confirmación está dada por las imágenes y la evolución clínica, la que generalmente es benigna. El antecedente de asma y consumo de cocaína es de utilidad para el diagnóstico definitivo. No requiere tratamiento quirúrgico y una vez descartados los diagnósticos diferenciales de gravedad, el paciente podría ser manejado en forma ambulatoria. La recidiva es infrecuente. El cuadro debe ser diagnóstico diferencial del dolor torácico agudo en especial en pacientes jóvenes.

5. Macia I, Moya J, Ramos R, Morera R, Escobar I, SAUMENCH J ET AL. Spontaneous pneumomediastinum: 41 cases. Eur J Cardio-Thorac Surg 2007; 31: 1110-4.

6. Campillo-Soto A, Coll-Salinas A, Soria-Aledo V, Blanco-Barrio A, Flores-Pastor B, Candel-Arenas M et al. Neumomediastino espontáneo: estudio descriptivo de nuestra experiencia basada en 36 casos. Arch Bronconeumol 2005; 41: 528-31.

7. Panacek EA, Singer AJ, Sherman BW, Prescott A, RutherFORD WF. Spontaneous pneumomediastinum: clinical and natural history. Ann Emerg Med 1992; 21: 1222-7. 
8. Newcomb AE, Clarke CP. Spontaneous pneumomediastinum: a benign curiosity or a significant problem? Chest 2005; 128: 3298-302.

9. Mondello B, Pavia R, Ruggeri P, Barone M, Barresi P, Monaco M. Spontaneous pneumomediastinum: experience in 18 adult patients. Lung 2007; 185: 9-14.

10. Jougon JB, Ballester M, Delcambre F, MacBride T, Dromer CE, Velly JF. Assessment of spontaneous pneumomediastinum: experience with 12 patients. Ann Thorac Surg 2003; 75: 1711-4.

11. Bodart E, De Bilderling G, Nisolle JF, Trigaux JP, TuerLinckX D. Spontaneous pneumomediastinum in an adolescent: an underestimated cause of chest pain. Arch Pediatr 1999; 6: 1293-6.

12. LAENNEC RTH. A treatise on diseases of the chest and mediate auscultation, translated by John Forbes, $2^{\text {nd }}$ Ed. London: T and G Underwood; 1827.

13. Simmons ST. A case of emphysema brought on by severe labor pains. Lond Med Commun 1783; 1: 176.

14. Golpe Gómez R, Mateos Colino A, Docasar Bertolo L, Miguélez Vara C. Spontaneous pneumomediastinum associated with anxiety state. An Med Interna 2002; 19: 378-9.

15. MACKLIN MT, MACKLIN CC. Malignant interstitial emphysema of the lungs and mediastinum as an important occult complication in many respiratory diseases and other conditions: an interpretation of the clinical literature in the light of laboratory experiment. Medicine 1944; 23: 281-358.

16. Maunder RJ, Pierson DJ, Hudson LD. Subcutaneous and mediastinal emphysema: pathophysiology, diagnosis and management. Arch Intern Med 1984; 144: $1447-53$.

17. Caramella D, Bulleri A, Battolla L, Pifferi M, Baldini G, Bartolozzi C. Spontaneous epidural emphysema and pneumomediastinum during an asthmatic attack in a child. Pediatr Radiol 1997; 27: 929-31.

18. Balachandran S, Guinto C, Goodman P. Epidural pneumatosis associated with spontaneous pneumomediastinum. AJNR 1993; 14: 271-2.

19. Ovalle P, Arce J. Neumomediastino espontáneo: enfisema retrofaríngeo forma de presentación no habitual. Rev Chil Radiol 2005; 11: 116-21.

20. Mir S, Korn O, Mora I, Berríos D. Neumomediastino espontáneo: a propósito de un caso. Rev Chil Cir 2007; 59: 459-62.

21. Freixinet J, García F, Rodríguez PM, Santana NB, Quintero CO, Hussein M. Spontaneous pneumomediastinum longterm follow-up. Respir Med 2005; 99: 1160-3.

22. Koullias GJ, Korkolis DP, Wang XJ, Hammond GL. Current assessment and management of spontaneous pneumomediastinum: experience in 24 adult patients. Eur J Cardiothorac Surg 2004; 25: 852-5.
23. O'Sullivan AJ, Casey JH. Spontaneous pneumomediastinum and diabetic ketoacidosis. Med J Aust 1997; 166: 245-6.

24. Weathers LS, Brooks WG, Declue TJ. Spontaneous pneumomediastinum in a patient with diabetic ketoacidosis: a potentially hidden complication. South Med J 1995; 88: 483-4.

25. Van Veelen I, Hogeman PH, Van Elburg A, NielsenAbbring FW, Heggelman BG, Mahieu HF. Pneumomediastinum: a rare complication of anorexia nervosa in children and adolescents. A case study and review of the literature. Eur J Pediatr 2007; [Epub ahead of print].

26. Donley AJ, Kemple TJ. Spontaneous pneumomediastinum complicating anorexia nervosa. Br Med J 1978; 2: 1604-5.

27. Raley JC, Andrews JI. Spontaneous pneumomediastinum presenting as jaw pain during labor. Obstet Gynecol 2001; 98: 904-6.

28. Norzilawati MN, Shuhaila A, Zainul Rashid MR. Postpartum pneumomediastinum. Singapore Med J 2007; 48: 174-6.

29. Le Floch as, Lapostolle F, Danhiez F, Adnet F. Pneumomediastinum as a complication of recreational ecstasy use. Ann Fr Anesth Reanim 2002; 21: 35-7.

30. Marasco SF, Lim HK. Ecstasy-associated pneumomediastinum. Ann R Coll Surg Engl 2007; 89: 389-93.

31. Maeder M, Ullmer E. Pneumomediastinum and Bilateral Pneumothorax as a Complication of Cocaine Smoking. Respiration 2003; 70: 407.

32. Haim DY, Lippmann ML, Goldberg SK, Walkenstein MD. The pulmonary complications of crack cocaine. A comprehensive review. Chest 1995; 107: 233-40.

33. Nemet D, Suchard JR, Dibernardo LM, Mukai DS, Cooper DM. Pneumomediastinum and subcutaneous emphysema after pulmonary function tests in a young healthy woman. Eur J Emerg Med 2004; 11: 105-7.

34. Minos P, Potaris K, Gakidis I, Mazaris E, Sarras E, Kontos Z. Sports-related spontaneous pneumomediastinum. Ann Thorac Surg 2004; 78: 983-6.

35. Dekel B, Paret G, Szeinberg A, Vardi A, Barzilay Z. Spontaneous pneumomediastinum in children: clinical and natural history. Eur J Pediatr 1996; 155: 695-7.

36. ABolnik I, Lossos IS, Breuer R. Spontaneous pneumomediastinum. A report of 25 cases. Chest 1991; 100; 93-5.

37. Zylak CM, Standen JR, Barnes GR, Zylak CJ. Pneumomediastinum revisited. RadioGraphics 2000; 20: 1043-57.

38. Martin MF, Hlaawatsch A, Heussel CP, Schwaden F, KAUCZOR HU. The radiologic findings in pneumomediastinum. Value of conventional radiography and comparison with computerized tomography. Radiologia 1997; 39: 709-14. 